\title{
PARAMETRIC RADIATION \\ https://doi.org/10.46813/2021-134-096 \\ TRANSITION EXCITATION OF A PERFECTLY CONDUCTING CYLINDER BY RELATIVISTIC ELECTRON BUNCHES
}

\author{
V.A. Balakirev, I.N. Onishchenko \\ National Science Center "Kharkov Institute of Physics and Technology”, Kharkiv, Ukraine \\ E-mail:vabalakirev@kipt.kharkov.ua
}

Transition radiation of a relativistic electron bunch, which arises when it collides with the surface of an infinite perfectly conducting cylinder, is considered. The electron bunch moves perpendicular to the cylinder surface. Expressions for the field strength of electromagnetic radiation in the wave zone are obtained and investigated.

PACS: 41.75.Lx, 41.85.Ja, 41.69.Bq

\section{INTRODUCTION}

Transition and diffraction radiation of relativistic charged particles is widely used in various fields of physics [1 - 4]. These elementary radiation processes can be used to obtain coherent electromagnetic radiation from centimeter to infrared wavelength ranges, depending on the size of electron bunches grouped in one way or another. We also note that the effect of transition radiation of high-current relativistic electron bunches can be used to obtain powerful ultra-broadband electromagnetic pulses [5 - 7].

In the present work, we study the transition radiation arising from the collision of a relativistic electron bunch with a perfectly conductive infinite cylinder for the case, when the electron bunch moves perpendicular to the surface of the cylinder. Expressions for the strength of electromagnetic radiation in the wave zone are obtained and investigated. The main attention is paid to the influence of the guiding properties of the perfectly conductive cylinder on the main characteristics of the transition radiation, first of all, the radiation pattern.

\section{STATEMENT OF THE PROBLEM. BASIC EQUATIONS}

Let's consider an infinitely long perfectly conductive round cylinder of radius $a$. The cylinder is in a vacuum. A relativistic electron bunch (or periodic sequence of bunches) moves in a vacuum toward the cylinder along a straight trajectory perpendicular to the cylinder surface. The problem is to determine the field of electromagnetic radiation in all space surrounding the cylinder and, ultimately, in the wave zone.

We will assume that the electron bunch infinitely thin in the transverse plane relative to the direction of its motion. In the longitudinal direction, the electron bunch has finite dimensions. Let us choose a cylindrical coordinate system, the axis $z$ of which coincides with the axis of the cylinder. Then the current density of a single bunch can be represented as

$$
\vec{j}=-\vec{e}_{r} \frac{Q}{t_{b}} \Pi\left(\frac{t+r / v_{0}}{t_{b}}\right) \frac{1}{r} \delta(\varphi) \delta(z),
$$

where $\vec{e}_{r}$ is the unit vector in the radial direction, $Q$ is the charge of the bunch, $v_{0}, t_{b}$ are its velocity and duration. The function $\Pi(\tau)$ describes the longitudinal pro- file of the bunch and satisfies the normalization condition

$$
\int_{-\infty}^{\infty} \Pi(\tau) d \tau=1
$$

$\delta(x)$ is delta-function.

For a periodic sequence of bunches for the current density, we have the sum of the currents of individual bunches

$$
\vec{j}=-\vec{e}_{r} \frac{Q}{t_{b}} \frac{1}{r} \delta(\varphi) \delta(z) \sum_{l=-\infty}^{\infty} \Pi\left(\frac{t-l T+r / v_{0}}{t_{b}}\right),
$$

$T$ is the bunch repetition period.

To solve Maxwell's equations, the current density of an individual bunch (1) is conveniently expanded in the Fourier integral

$$
\vec{j}=-\vec{e}_{r} \int_{-\infty}^{\infty} j_{r \omega}(r, z, \varphi) e^{-i \omega t} d \omega,
$$

where

$$
\begin{aligned}
& j_{r \omega}(r, z, \varphi)=Q \Pi_{\omega} \frac{e^{-i k_{b} r}}{r} \delta(\varphi) \delta(z), \\
& \Pi_{\omega}=\frac{1}{2 \pi} \int_{-\infty}^{\infty} \Pi(\tau) e^{i \omega t_{b} \tau} d \tau, k_{b}=\omega / v_{0} .
\end{aligned}
$$

For a periodic sequence of bunches, the current density (2) should be represented as a Fourier series

$$
\vec{j}=-\vec{e}_{r} \sum_{l=-\infty}^{\infty} j_{r l}(r, z, \varphi) e^{-i \omega_{l} t}
$$

Here

$$
\begin{aligned}
& j_{r l}(r, z, \varphi)=\frac{Q}{t_{b}} \Pi_{l} \frac{e^{-i l k_{m} r}}{r} \delta(\varphi) \delta(z), \\
& \Pi_{l}=\sum_{l^{\prime}=-\infty}^{\infty} \frac{1}{T} \int_{-T / 2}^{T / 2} e^{i l \omega_{m} t} \Pi\left(\frac{t-l^{\prime} T}{t_{b}}\right) d t,
\end{aligned}
$$

$k_{m}=\omega_{m} / v_{0}, \omega_{m}=2 \pi / T$.

If the bunches do not overlap within the repetition period, then in the sum (6) it is sufficient to take into account only one term $l^{\prime}=0$

$$
\Pi_{l}=\frac{1}{T} \int_{-T / 2}^{T / 2} e^{i l \omega_{m} t} \Pi\left(\frac{t}{t_{b}}\right) d t
$$

Accordingly, the electromagnetic field must also be expanded either in the integral

$$
(\vec{E}, \vec{H})=\int_{-\infty}^{\infty}\left(\vec{E}_{\omega}, \vec{H}_{\omega}\right) e^{-i \omega t} d \omega,
$$


or in the Fourier series

$$
(\vec{E}, \vec{H})=\sum_{l=-\infty}^{\infty}\left(\vec{E}_{l}, \vec{H}_{l}\right) e^{-i l \omega_{m} t}
$$

Due to the geometry of the system, the electric field of the electron bunch will induce longitudinal and azimuthal surface currents on the cylinder surface. In turn, the longitudinal surface current will excite the $E$ components of the electromagnetic field, and the azimuthal surface current will excite the $H$-components. Thus, in the considered system, a radial currents of the form (1) or (2) will excite all six components of the electromagnetic field.

In cylindrical geometry, it is convenient to start from the equations for the longitudinal Fourier components of the electric $E_{\omega z}$ and magnetic $H_{\omega z}$ fields

$$
\begin{gathered}
\Delta E_{z \omega}+k_{0}^{2} E_{z \omega}=\frac{4 \pi i}{c k_{0}} \frac{1}{r} \frac{\partial}{\partial r} r \frac{\partial j_{r \omega}}{\partial z}, \\
\Delta H_{z \omega}+k_{0}^{2} H_{z \omega}=-\frac{4 \pi}{c} \frac{1}{r} \frac{\partial j_{r \omega}}{\partial \varphi} .
\end{gathered}
$$

The other field components are related with components $E_{\omega z}$ and $H_{\omega z}$ by the equations

$$
\begin{aligned}
& \left(\frac{\partial^{2}}{\partial z^{2}}+k_{0}^{2}\right) E_{r \omega}=i k_{0} \frac{1}{r} \frac{\partial H_{z \omega}}{\partial \varphi}+\frac{\partial^{2} E_{z \omega}}{\partial r \partial z}+\frac{4 \pi}{c} i k_{0} j_{r \omega}, \\
& \left(\frac{\partial^{2}}{\partial z^{2}}+k_{0}^{2}\right) H_{\varphi \omega}=i k_{0} \frac{\partial E_{z \omega}}{\partial r}+\frac{1}{r} \frac{\partial^{2} H_{z \omega}}{\partial \varphi \partial z}+\frac{4 \pi}{c} \frac{\partial j_{r \omega}}{\partial z}, \\
& \left(\frac{\partial^{2}}{\partial z^{2}}+k_{0}^{2}\right) H_{r \omega}=\frac{\partial^{2} H_{z \omega}}{\partial r \partial z}-i k_{0} \frac{1}{r} \frac{\partial E_{z \omega}}{\partial \varphi}, \\
& \left(\frac{\partial^{2}}{\partial z^{2}}+k_{0}^{2}\right) E_{\varphi \omega}=-i k_{0} \frac{\partial H_{z \omega}}{\partial r}+\frac{1}{r} \frac{\partial^{2} E_{z \omega}}{\partial \varphi \partial z}, \\
& k_{0}=\omega / c .
\end{aligned}
$$

The system of equations must be supplemented with boundary conditions, which consist in the vanishing of the tangential components of the electric field on the surface of the cylinder and the condition of radiation at infinity in free space.

We represent the Fourier amplitude of the current density $j_{r \omega}(r, \varphi, z)$ as a combination of the series and the Fourier integral

$$
j_{r \omega}=\sum_{n=-\infty}^{\infty} e^{i n \varphi} \int_{-\infty}^{\infty} j_{r \omega n k}(r) e^{i k z} d k,
$$

where

$$
j_{r \omega n k}(r)=\frac{1}{(2 \pi)^{2}} \int_{0}^{2 \pi} d \varphi e^{-i n \varphi} \int_{-\infty}^{\infty} j_{r \omega}(\varphi, z, r) e^{-i k z} d z .
$$

For the Fourier amplitude of the current density (4), we obtain

$$
j_{r \omega n k}(r)=\frac{1}{(2 \pi)^{2}} Q \Pi_{\omega} \frac{e^{-i k_{b} r}}{r} .
$$

In the case of a periodic sequence of relativistic electron bunches, instead of (12), we have

$$
j_{r l n k}(r)=\frac{1}{(2 \pi)^{2}} \frac{Q}{t_{b}} \Pi_{l} \frac{e^{-i l k_{m} r}}{r} .
$$

The components of the electromagnetic field described by the system of equations (9) - (11) must be represented in the same form

$$
\left(\vec{E}_{\omega}, \vec{H}_{\omega}\right)=\sum_{n=-\infty}^{\infty} e^{i n \varphi} \int_{-\infty}^{\infty}\left(\vec{E}_{\omega n k}, \vec{H}_{\omega n k}\right) e^{i k z} d k .
$$

Then, instead of partial differential equations (9), (10), we obtain simpler ordinary differential equations for the amplitudes of the expansion of the longitudinal components of the electromagnetic field (13)

$$
\begin{aligned}
& \frac{1}{r} \frac{d}{d r} r \frac{d E_{z \alpha}}{d r}+\left(v^{2}-\frac{n^{2}}{r^{2}}\right) E_{z \alpha}=\frac{4 \pi k}{c k_{0}} \frac{1}{r} \frac{d}{d r} r j_{r \alpha}(r), \\
& \frac{1}{r} \frac{d}{d r} r \frac{d H_{z \alpha}}{d r}+\left(v^{2}-\frac{n^{2}}{r^{2}}\right) H_{z \alpha}=\frac{4 \pi}{c} \frac{i n}{r} j_{r \alpha}(r),
\end{aligned}
$$

where subscript $\alpha$ denotes a group of indices $\omega n k$. The transverse components of the electromagnetic field are related to the longitudinal components by the relations

$$
\begin{aligned}
& E_{r \alpha}=-\frac{k_{0}}{v^{2}} \frac{n}{r} H_{z \alpha}+i \frac{k}{v^{2}} \frac{d E_{z \alpha}}{d r}+\frac{4 \pi}{c} \frac{i k_{0}}{v^{2}} j_{r \alpha}, \\
& H_{\varphi \alpha}=-\frac{k}{v^{2}} \frac{n}{r} H_{z \alpha}+i \frac{k_{0}}{v^{2}} \frac{d E_{z \alpha}}{d r}+\frac{4 \pi}{c} \frac{i k}{v^{2}} j_{r \alpha}, \\
& H_{r \alpha}=\frac{k_{0}}{v^{2}} \frac{n}{r} E_{z \alpha}+i \frac{k}{v^{2}} \frac{d H_{z \alpha}}{d r} \\
& E_{\varphi \alpha}=-\frac{k}{v^{2}} \frac{n}{r} E_{z \alpha}-i \frac{k_{0}}{v^{2}} \frac{d H_{z \alpha}}{d r}
\end{aligned}
$$

where $v^{2}=k_{0}^{2}-k^{2}$.

On the surface of a perfectly conductive cylinder, the following conditions must be satisfied

$$
E_{z \alpha}(r=a)=E_{\varphi \alpha}(r=a)=0 .
$$

The radiation condition (absence of waves coming from infinity) can be written as follows

$$
\vec{E}_{\alpha}(r \rightarrow \infty), \vec{H}_{\alpha}(r \rightarrow \infty) \sim e^{i v r} .
$$

Solutions of second-order inhomogeneous differential equations (14), (15) satisfying the indicated conditions for electromagnetic field have the form

$$
\begin{gathered}
E_{z \alpha}=-2 i \pi^{2} \frac{k}{c k_{0}} \frac{1}{H_{n}^{(1)}(v a)}\left[\Delta_{n}(v r, v a) \int_{r}^{\infty} H_{n}^{(1)^{\prime}}(v r) j_{r \alpha}(r) r d r+\right. \\
\left.+H_{n}^{(1)}(v r) \int_{a}^{r} \Delta_{n}^{\prime}(v r, v a) j_{r \alpha}(r) r d r\right] \\
H_{z \alpha}=-2 \pi^{2} \frac{n}{c} \frac{1}{H_{n}^{(1)^{\prime}}(v a)}\left[\Delta_{n}^{(1)}(v r, v a) \int_{r}^{\infty} H_{n}^{(1)}(v r) j_{r \alpha}(r) d r+\right. \\
\left.+H_{n}^{(1)}(v r) \int_{a}^{r} \Delta_{n}^{(1)}(v r, v a) j_{r \alpha}(r) d r\right]
\end{gathered}
$$

Here $F_{n}^{\prime}(v r) \equiv d F_{n}(v r) / d r$

$$
\begin{gathered}
\Delta_{n}(v r, v a)=H_{n}^{(1)}(v r) J_{n}(v a)-H_{n}^{(1)}(v a) J_{n}(v r), \\
\Delta_{n}^{(1)}(v r, v a)=H_{n}^{(1)}(v r) J_{n}^{\prime}(v a)-H_{n}^{(1)^{\prime}}(v a) J_{n}(v r) .
\end{gathered}
$$

Expressions for the transverse components of the electromagnetic field follow from relations (16).

\section{FIELD OF TRANSITION RADIATION IN THE WAVE ZONE}

The field of electromagnetic radiation in the wave zone is of the greatest interest. To determine it in total expressions for the longitudinal field components (18), (19) it is necessary by passing to the limit $r \rightarrow \infty$, to 
select that part of the field which satisfies the radiation condition (17). As a result, we obtain

$$
\begin{gathered}
E_{z \alpha}^{(r a d)}=-2 i \pi^{2} \frac{k}{c k_{0}} \frac{H_{n}^{(1)}(v r)}{H_{n}^{(1)}(v a)} \int_{a}^{\infty} \Delta_{n}^{\prime}(v r, v a) j_{r \alpha}(r) r d r, \\
H_{z \alpha}^{(r a d)}=-2 \pi^{2} \frac{n}{c} \frac{H_{n}^{(1)}(v r)}{H_{n}^{(1)^{\prime}}(v a)} \int_{a}^{\infty} \Delta_{n}^{(1)}(v r, v a) j_{r \alpha}(r) d r .
\end{gathered}
$$

It is more preferable to perform further analysis for the azimuthal components of electromagnetic field. We find expressions for them from relations (16b), (16d)

$$
\begin{aligned}
& H_{\varphi \alpha}^{(r a d)}=2 \pi^{2} \frac{k}{c v^{2}} \frac{H_{n}^{(1)^{\prime}}(v r)}{H_{n}^{(1)}(v a)} \int_{a}^{\infty} \Delta_{n}^{\prime}(v r, v a) j_{r \alpha}(r) r d r, \\
& E_{\varphi \alpha}^{(r a d)}=2 i \pi^{2} \frac{k_{0} n}{c v^{2}} \frac{H_{n}^{(1)^{\prime}}(v r)}{H_{n}^{(1) \prime}(v a)} \int_{a}^{\infty} \Delta_{n}^{(1)}(v r, v a) j_{r \alpha}(r) d r .
\end{aligned}
$$

And, finally, taking into account the combined expansion (13), we obtain integral representations for the frequency Fourier-amplitudes of the azimuthal components of the radiation field

$$
\begin{gathered}
H_{\varphi \omega}^{(r a d)}=\frac{2 \pi^{2}}{c}\left[H_{\omega 0}(r, z)+2 \sum_{n=1}^{\infty} H_{\omega n}(r, z) \cos n \varphi\right], \\
H_{\omega n}(r, z)=\int_{-\infty}^{\infty} \frac{k}{v^{2}} \frac{S_{n}^{(h)}(v a)}{H_{n}^{(1)}(v a)} H_{n}^{(1)^{\prime}}(v r) e^{i k z} d k, \\
S_{n}^{(h)}(v a)=\int_{a}^{\infty} \Delta_{n}^{\prime}(v r, v a) j_{r \alpha}(r) r d r, \\
E_{\varphi \omega}^{(r a d)}(r, z)=-\frac{4 \pi^{2} k_{0}}{c} \sum_{n=1}^{\infty} n E_{\omega n}(r, z) \sin n \varphi, \\
E_{\omega n}(r, z)=\int_{-\infty}^{\infty} \frac{1}{v^{2}} \frac{S_{n}^{(e)}(v a)}{H_{n}^{(1)^{\prime}}(v a)} H_{n}^{(1)^{\prime}}(v r) e^{i k z} d k, \\
S_{n}^{(e)}(v a)=\int_{a}^{\infty} \Delta_{n}^{(1)}(v r, v a) j_{r \alpha}(r) d r .
\end{gathered}
$$

Note that $H_{\omega n}(r, z)=H_{\omega-n}(r, z) \quad$ and $E_{\omega n}(r, z)=E_{\omega-n}(r, z)$. The next step in the theory is the approximate calculation of integrals (21), (24). To do this, we will use the saddle-point method. Integrals of this type are well known in electrodynamics [8]. Therefore, we present the final expressions for the components of the electromagnetic field (20), (23)

$$
\begin{gathered}
H_{\varphi \omega}^{(r a d)}=i \frac{Q}{c} \frac{e^{i k_{0} R}}{R} \frac{\Pi_{\omega}}{\operatorname{tg} \vartheta}\left[L_{0}(\vartheta)+\right. \\
\left.+2 \sum_{n=1}^{\infty} L_{n}(\vartheta) e^{-i n \pi / 2} \cos n \varphi\right], \\
L_{n}(\vartheta)=\int_{\kappa_{0}}^{\infty} J_{n}(\rho \sin \vartheta) e^{-i \rho / \beta_{0}} d \rho- \\
-\frac{J_{n}\left(\kappa_{0} \sin \vartheta\right)}{H_{n}^{(1)}\left(\kappa_{0} \sin \vartheta\right)} \int_{\kappa_{0}}^{\infty} H_{n}^{(1)}(\rho \sin \vartheta) e^{-i \rho / \beta_{0}} d \rho, \\
E_{\varphi \omega}^{(r a d)}=-\frac{Q}{c} \frac{e^{i k_{0} R}}{R} \frac{\Pi_{\omega}}{\sin \vartheta} \sum_{n=1}^{\infty} n M_{n}(\vartheta) e^{-i n \pi / 2} \sin n \varphi, \\
M_{n}(\vartheta)=\int_{\kappa_{0}}^{\infty} J_{n}(\rho \sin \vartheta) \frac{e^{-i \rho / \beta_{0}}}{\rho} d \rho-
\end{gathered}
$$

$\kappa_{0}=k_{0} a$.

$$
-\frac{J_{n}^{\prime}\left(\kappa_{0} \sin \vartheta\right)}{H_{n}^{(1)^{\prime}}\left(\kappa_{0} \sin \vartheta\right)} \int_{\kappa_{0}}^{\infty} H_{n}^{(1)}(\rho \sin \vartheta) \frac{e^{-i \rho / \beta_{0}}}{\rho} d \rho,
$$

First of all, we note that the $H$-components of the electromagnetic radiation field $\left(H_{z}, H_{r}, E_{\varphi}\right)$ contain only asymmetric modes $n \neq 0$. There is no symmetrical mode $n=0$. As for the $E$-components $\left(E_{z}, E_{r}, H_{\varphi}\right)$ of the field, they contain both symmetric and asymmetric modes.

Consider a conductive cylinder with a small radius $\omega a / c \ll 1$ or a low-frequency range of the transition radiation spectrum. Transition radiation is mainly concentrated in the specified frequency range for electron bunches with duration $t_{b} \gg a / c$. Under these conditions, waves with higher frequencies are radiated weakly, since for waves with frequencies $\omega \gg c / a$, the condition of the radiation coherence by an electron bunch is violated.

Let us first of all consider the asymptotic representation of integral (27) in the considered limiting case $\kappa_{0}=k_{0} a \ll 1$ for a symmetric mode $n=0$. The expression for the coefficient $L_{0}$ can be written as follows

$$
\begin{gathered}
L_{0}(\vartheta)=\int_{0}^{\infty} J_{0}(\rho \sin \vartheta) e^{-i \rho / \beta_{0}} d \rho+ \\
+\frac{\pi}{2} \frac{1}{\ln \left(2 / v \kappa_{0} \sin \vartheta\right)} \int_{0}^{\infty} N_{0}(\rho \sin \vartheta) e^{-i \rho / \beta_{0}} d \rho,
\end{gathered}
$$

$N_{0}(x)$ is the Neumann function, $v$ is the Euler constant. In expression (27), we used the asymptotic representations of cylindrical functions for small values of the argument, and in the integrals we installed the lower limit equal to zero. The integrals included in (30) are tabular [9]. After substitution their values into (30), we obtain the final expression for the coefficient $L_{0}$

$$
L_{0}(\vartheta)=-i \beta_{0} \gamma(\vartheta) \frac{\ln \frac{2 \beta_{0} \gamma(\vartheta)}{v \kappa_{0}(\gamma(\vartheta)+1)}}{\ln \left(2 / v \kappa_{0} \sin \vartheta\right)},
$$

where

$$
\gamma(\vartheta)=\frac{1}{\sqrt{1-\beta_{0}^{2} \sin ^{2} \vartheta}} .
$$

Let us now consider the coefficients $L_{n}$ for asymmetric modes. Here we also use the asymptotic representations for cylindrical functions of order $n \geq 1$. As a result, we obtain

$$
\begin{gathered}
L_{n}(\vartheta)=\int_{0}^{\infty} J_{n}(\rho \sin \vartheta) e^{-i \rho / \beta_{0}} d \rho- \\
-\frac{\pi}{n \Gamma^{2}(n)}\left(\frac{\kappa_{0} \sin \vartheta}{2}\right)^{2 n} \int_{\kappa_{0}}^{\infty} N_{n}(\rho \sin \vartheta) e^{-i \rho / \beta_{0}} d \rho .
\end{gathered}
$$

The first integral in (32) is also tabular. As for the second integral, it should be taken into account that the main contribution to the value of the integral gives the region of small $\rho$. Therefore, to estimate it, one can use the asymptote of the Neumann function for small values of the argument. As a result, taking into account 
the above, we obtain the following approximate expression for the coefficients $L_{n}$

$$
\begin{gathered}
L_{n}(\vartheta)=-i \beta_{0} \gamma(\vartheta) e^{-i \pi n / 2}\left(\frac{\beta_{0} \gamma(\vartheta) \sin \vartheta}{\gamma(\vartheta)+1}\right)^{n}+\Delta_{n}, \\
\Delta_{n}= \begin{cases}-\frac{\kappa_{0} \sin \vartheta}{2} \kappa_{0} \ln \frac{1}{\kappa_{0}}, & n=1 ; \\
\frac{\kappa_{0}}{(n-1) \Gamma(n+1)}\left(\frac{\kappa_{0} \sin \vartheta}{2}\right)^{n}, & n>1 .\end{cases}
\end{gathered}
$$

Now let's move on to calculating the coefficients $M_{n}(\vartheta)$. In the considered approximation $\kappa_{0}=k_{0} a \ll 1$, the expression for them (29) can be simplified and represented in the form

$$
\begin{gathered}
M_{n}(\vartheta)=\int_{0}^{\infty} J_{n}(\rho \sin \vartheta) \frac{e^{-i \rho / \beta_{0}}}{\rho} d \rho+ \\
+\frac{\pi}{\Gamma(n) \Gamma(n)}\left(\frac{\kappa_{0} \sin \vartheta}{2}\right)^{2 n} \int_{\kappa_{0}}^{\infty} N_{n}(\rho \sin \vartheta) \frac{e^{-i \rho / \beta_{0}}}{\rho} d \rho .
\end{gathered}
$$

The first integral is tabular. The second integral can be estimated approximately. As a result, we obtain the following expression for the expansion coefficients

$$
\begin{gathered}
M_{n}(\vartheta)=\frac{e^{-i \pi n / 2}}{n}\left(\frac{\beta_{0} \gamma(\vartheta) \sin \vartheta}{\gamma(\vartheta)+1}\right)^{n}+D_{n}, \\
D_{n}=-\frac{1}{n \Gamma(n+1)}\left(\frac{\kappa_{0} \sin \vartheta}{2}\right)^{n}, n \geq 1 .
\end{gathered}
$$

We can be seen that the terms $\Delta_{n}$ and $D_{n}$ are small and rapidly decrease with increasing number $n$. Therefore, they can be neglected. Taking into account the above, expressions for the azimuthal components of the field (26), (28) take the form

$$
H_{\varphi \omega}^{(\mathrm{rad})}=\frac{Q}{c \beta_{0}} \Pi_{\omega} \frac{e^{i k_{0} R}}{R}\left[F_{s}(\omega, \vartheta)+F_{a s}(\vartheta, \varphi)\right],
$$

where

$$
\begin{gathered}
F_{s}(\omega, \vartheta)=\frac{\beta_{0} \gamma(\vartheta) \cos \vartheta}{\sin \vartheta} \frac{\ln \frac{2 \beta_{0} \gamma(\vartheta)}{v \kappa_{0}(\gamma(\vartheta)+1)}}{\ln \left(2 / v \kappa_{0} \sin \vartheta\right)}, \\
F_{a s}(\vartheta, \varphi)=\frac{2 \beta_{0} c t g \vartheta}{\sqrt{1-\beta_{0}^{2} \sin ^{2} \vartheta}} \sum_{n=1}^{\infty}\left[e^{-i \pi} Q(\vartheta)\right]^{n} \cos n \varphi, \\
E_{\varphi \omega}^{(r a d)}=-\frac{Q}{c} \Pi_{\omega} \frac{e^{i k_{0} R}}{R} G_{a s}(\vartheta, \varphi), \\
G_{a s}(\vartheta, \varphi)=\frac{2}{\sin \vartheta} \sum_{n=1}^{\infty}\left[e^{-i \pi} Q(\vartheta)\right]^{n} \sin n \varphi, \\
Q(\vartheta)=\frac{\beta_{0} \gamma(\vartheta) \sin \vartheta}{\gamma(\vartheta)+1} .
\end{gathered}
$$

The series included in expressions (37) and (39) are summed exactly. As a result, for the functions $F_{a s}(\vartheta, \varphi)$ and $G_{a s}(\vartheta, \varphi)$ we obtain the following expressions

$$
\begin{gathered}
F_{a s}(\vartheta, \varphi)=-2 \frac{Q(\vartheta) \gamma(\vartheta)}{\operatorname{tg} \vartheta} \frac{\cos \varphi+Q(\vartheta)}{[\cos \varphi+Q(\vartheta)]^{2}+\sin ^{2} \varphi}, \\
G_{a s}(\vartheta, \varphi)=-\frac{2 \beta_{0} \gamma(\vartheta)}{\gamma(\vartheta)+1} \frac{\sin \varphi}{[\cos \varphi+Q(\vartheta)]^{2}+\sin ^{2} \varphi} .
\end{gathered}
$$

The function $F_{s}(\vartheta)$ describes the dependence of the symmetric part of the azimuthal component of the magnetic field $H_{\varphi \omega}^{(r a d)}$ on the polar angle $\vartheta$, and the function $F_{a s}(\vartheta, \varphi)$ describes asymmetric part. In the azimuthal component of the electric field, the symmetrical part is absent.

Let note that the function $F_{s}(\vartheta)$ has a singularity for the polar angles $\vartheta=0$ and $\vartheta=\pi$. For these angles, the function and, consequently, the radiation field along the conductor becomes infinity. An asymmetric field does not have such a feature. A cylindrical conductor has a guiding effect on a symmetrical wave. Note that the guiding action of a cylindrical conductor is also manifested during wave diffraction [10] and excitation of cylindrical conductors by concentrated currents. The field singularity is integrable, so the total radiated energy remains finite. In fact, in this situation, a thin cylindrical conductor is a traveling wave antenna excited by a relativistic electron bunch. Accordingly, the radiation pattern of symmetric radiation is determined primarily by the antenna geometry and weakly depends on the relativistic factor of the bunch.

Let us show that the asymmetric terms in the expressions for the azimuthal field components (35), (38) describe the transition radiation, which is characterized by a narrow radiation pattern in the region of small angles relative to the direction of the bunch motion. Let us consider the dependence of the electromagnetic field components of radiation on the angles $\vartheta$ and $\varphi$. It is convenient to perform replacement $\vartheta=\pi / 2+\bar{\vartheta}$, $\varphi=\pi+\bar{\varphi}$. For the new angles, the expressions for the functions $F_{a s}(\vartheta, \varphi)$ and $\varphi=\pi+\bar{\varphi}$ take the form

$$
\begin{aligned}
F_{a s}(\bar{\vartheta}, \bar{\varphi})= & 2 \bar{\gamma}(\vartheta) \bar{Q}(\vartheta) \frac{\operatorname{tg} \bar{\vartheta}[\bar{Q}(\bar{\vartheta})-\cos \bar{\varphi}]}{[\bar{Q}(\bar{\vartheta})-\cos \bar{\varphi}]^{2}+\sin ^{2} \bar{\varphi}}, \\
G_{a s}(\bar{\vartheta}, \bar{\varphi})= & -\frac{2 \beta_{0} \bar{\gamma}(\vartheta)}{\bar{\gamma}(\vartheta)+1} \frac{\sin \bar{\varphi}}{[\bar{Q}(\bar{\vartheta})-\cos \bar{\varphi}]^{2}+\sin ^{2} \bar{\varphi}}, \\
\bar{\gamma}(\bar{\vartheta})= & \frac{1}{\sqrt{1-\beta_{0}^{2} \cos ^{2} \bar{\vartheta}}}, \bar{Q}(\vartheta)=\frac{\beta_{0} \bar{\gamma}(\bar{\vartheta}) \cos \bar{\vartheta}}{\bar{\gamma}(\bar{\vartheta})+1} \\
& \pi / 2>\bar{\vartheta}>-\pi / 2, \pi>\bar{\varphi}>-\pi
\end{aligned}
$$

First of all, we note that in the plane $\bar{\vartheta}=0 \quad(z=0)$, in which the electron bunch propagates, the function $F_{a s}(0, \bar{\varphi})=0$, i.e. there is no radiation of $E$ electromagnetic waves in this plane. Let's consider the radiation pattern in the plane $\bar{\varphi}=0$ or $\varphi=\pi$. For this value of the azimuthal angle, the function $F_{a s}(\bar{\vartheta}, 0)$ takes the form

$$
F_{a s}(\bar{\vartheta}, 0)=-\frac{2 \beta_{0} \sin \bar{\vartheta}}{1-\beta_{0} \cos \bar{\vartheta}} \frac{\gamma(\bar{\vartheta})}{\left(1+\beta_{0} \cos \bar{\vartheta}\right) \gamma(\bar{\vartheta})+1} .
$$

For small polar angles with respect to the direction of the relativistic bunch motion $\bar{\vartheta} \ll 1$, this expression is simplified

$$
F_{a s}(\bar{\vartheta}, 0)=-\frac{2 \bar{\vartheta}}{\bar{\vartheta}^{2}+\gamma_{0}^{-2}},
$$


where $\gamma_{0}$ is the relativistic factor of the bunch electrons. In the directions

$$
\bar{\vartheta}= \pm 1 / \gamma_{0}
$$

$\left|F_{a s}(\bar{\vartheta}, 0)\right|$, and, consequently, the transition radiation field has a sharp maximum by polar angle $\bar{\vartheta}$

$$
\left|F_{a s}(\bar{\vartheta}, 0)\right|_{\max }=\gamma_{0}>>1 \text {. }
$$

Expressions (44), (45) are valid in a narrow range of the azimuthal angle

$$
|\bar{\varphi}| \ll \gamma_{0}^{-2}
$$

Outside this interval of the azimuthal angle, the radiation of $E$-electromagnetic waves loses its narrow directivity.

Let us now investigate expression (43), which describes the dependence of the $H$-components of the field of electromagnetic radiation on the angular coordinates. For further analysis, it is convenient to make some transformation of the function $G_{a s}(\bar{\vartheta}, \bar{\varphi})$ and represent it in the form

$$
\begin{gathered}
G_{a s}(\bar{\vartheta}, \bar{\varphi})=-\frac{\beta(\bar{\vartheta})}{\cos \bar{\vartheta}} g_{a s}(\bar{\vartheta}, \bar{\varphi}), \\
g_{a s}(\bar{\vartheta}, \bar{\varphi}) \frac{\sin \bar{\varphi}}{1-\beta(\bar{\vartheta}) \cos \bar{\varphi}}, \\
\beta(\bar{\vartheta})=\frac{2 \bar{Q}(\bar{\vartheta})}{\bar{Q}^{2}(\bar{\vartheta})+1}<1, \bar{Q}=\frac{\beta_{0} \gamma(\bar{\vartheta}) \cos \bar{\vartheta}}{1+\gamma(\bar{\vartheta})}>1 .
\end{gathered}
$$

For the value of the azimuth angle

$$
\bar{\varphi}(\bar{\vartheta})= \pm \arcsin \frac{1}{\Gamma(\bar{\vartheta})}, \Gamma(\bar{\vartheta})=\frac{1+\bar{Q}^{2}(\bar{\vartheta})}{1-\bar{Q}^{2}(\bar{\vartheta})}>1
$$

function (43) reaches its maximum value $g_{a s}(\bar{\vartheta})=\Gamma(\bar{\vartheta})$, which depends only on the polar angle $\bar{\vartheta}$. In turn the function $\Gamma(\bar{\vartheta})$ is maximal at $\bar{\vartheta}=0$ and with increasing angle $\bar{\vartheta}$ decreases monotonically. In particular at $\bar{\vartheta}<<1$ for the function $\Gamma(\bar{\vartheta})$ we have the expression

$$
\Gamma(\bar{\vartheta})=\frac{\gamma_{0}}{\sqrt{1+\gamma_{0}^{2} \bar{\vartheta}^{2}}} .
$$

Thus, the sharpest radiation pattern of $H$ electromagnetic radiation is formed for angles

$$
\bar{\vartheta}^{2} \ll \gamma_{0}^{-2} \text {. }
$$

On the whole, the picture of asymmetric transition radiation looks as follows. The excited $E$-field forms on the full radiation pattern two sharp peaks with angles $\bar{\vartheta}= \pm 1 / \gamma_{0}$ in the horizontal plane $\bar{\varphi}=0$. For its part, the $H$-field forms two sharp peaks with angles $\bar{\varphi}= \pm 1 / \gamma_{0}$ on the radiation pattern in the plane $\bar{\vartheta}=0(z=0)$ perpendicular to the cylinder axis. Four maxima in the directional radiation pattern have the form of a cross in the plane perpendicular to the direction of motion of the bunch.

Thus, asymmetric transition radiation has a narrow directivity in directions close to the direction of motion of a relativistic electron bunch.

All that has been said above also fully applies to the case of excitation of a conductive cylinder by a periodic sequence of relativistic electron bunches. The only dif- ference is that in the latter case, field (7) with a discrete frequency spectrum $\omega=l \omega_{m}$ is excited.

A single bunch excites an electromagnetic field with a continuous frequency spectrum, and the pulse shape is determined by the Fourier integral (7). First of all, we note that the angular functions (40), (41) of the Fourier amplitudes of the asymmetric transition electromagnetic field do not depend on the frequency. Therefore, the shape of an asymmetric transition pulse, which is radiated at small angles to the direction of motion of the relativistic electron bunch, completely repeats the shape of the bunch [5]

$$
\begin{aligned}
& H_{\varphi}^{(a s)}=\frac{Q}{c t_{b}} \frac{F_{a s}(\bar{\vartheta}, \bar{\varphi})}{R} \Pi\left(\frac{t-R / c}{t_{b}}\right), \\
& E_{\varphi}^{(a s)}=\frac{Q}{c t_{b}} \frac{G_{a s}(\bar{\vartheta}, \bar{\varphi})}{R} \Pi\left(\frac{t-R / c}{t_{b}}\right) .
\end{aligned}
$$

In the case of a periodic sequence of relativistic electron bunches, the transition radiation will have the form of a periodic sequence of electromagnetic videopulses

$$
\begin{aligned}
& H_{\varphi}^{(a s)}=\frac{Q}{c t_{b}} \frac{F_{a s}(\vartheta, \varphi)}{R} \sum_{l=-\infty}^{\infty} \Pi\left(\frac{t-l T-R / c}{t_{b}}\right), \\
& E_{\varphi}^{(a s)}=\frac{Q}{c t_{b}} \frac{G_{a s}(\vartheta, \varphi)}{R} \sum_{l=-\infty}^{\infty} \Pi\left(\frac{t-l T-R / c}{t_{b}}\right) .
\end{aligned}
$$

The symmetric angular function $F_{s}(\omega, \vartheta)$ in general case depends on frequency. Therefore, the shape of the symmetric transition pulse will differ from the profile of the electron bunch. However, as follows from expression (36) for polar angles

$$
\ln \left(1 / k_{0} a\right) \sim \ln \left(c t_{b} / a\right)>>\ln (1 / \sin \vartheta)
$$

or

$$
\sin \vartheta>a / c t_{b}<<1
$$

there is practically no dependence of the angular function on frequency

$$
F_{s}(\omega, \vartheta)=\frac{\beta_{0} \cos \vartheta}{\sin \vartheta \sqrt{1-\beta_{0}^{2} \sin ^{2} \vartheta}} .
$$

In this case, the shape of the symmetrical transition video pulse will also repeat the profile of the electron bunch. The magnetic field of a symmetric transition pulse is described by formula (48), in which $F_{a s}(\bar{\vartheta}, \bar{\varphi})$ should be replaced by (50). If the condition (49) is not satisfied, then it is necessary to take into account the dependence of the angular function on frequency

$$
F_{s}(\omega, \vartheta)=\frac{\ln \frac{c}{v \omega a}}{\vartheta \ln \frac{2 c}{v \omega a \vartheta}} .
$$

In this case, the shape of the radiated pulse differs from the bunch profile.

\section{CONCLUSIONS}

Transition radiation of a relativistic electron bunch, which arises when it collides with the surface of an infinite ideally conductive cylinder, is considered. The electron bunch moves perpendicular to the cylinder surface. Expressions for the strength of electromagnetic radiation in the wave zone are obtained. It is shown that this 
expression contains two terms. The first term describes the actual transition electromagnetic radiation of the electron bunch. This radiation is azimuthally asymmetric and is primarily determined by the properties and parameters of the electron bunch itself. The radiation contains both $E$ - and $H$-field components and is directed at a small angle to the direction of the bunch motion. The radiation angle is inversely proportional to the relativistic factor of the bunch, and the strength of the electric and magnetic field in this direction is proportional to the relativistic factor. The second term describes the radiation of the current induced on the surface of a perfectly conductive cylinder and propagating along the cylinder at the speed of light in vacuum. The strength of this field has an integrable singularity (turns to infinity) strictly along the surface of the cylinder. The peculiarity is due to the guiding properties of a perfectly conducting cylinder.

The spatial structure (radiation pattern) of this transition electromagnetic radiation weakly depends on the parameters (energy) of the bunch, and is mainly determined by the geometry of the considered cylindrical antenna. The shape of the radiated electromagnetic pulse has been determined.

In the case of a periodic sequence of relativistic electron bunches, the transition radiation will have the form of a periodic sequence of electromagnetic pulses.

\section{REFERENCES}

1. V.L. Ginzburg, V.N. Tsytovich. Transition radiation and transition scattering. Moscow: "Nauka", 1984.
2. B.M. Bolotovsky, G.V. Voskresensky. Diffraction radiation // UFN. 1966, v. 88, issue 2, p. 209-251.

3. B.M. Bolotovsky, E.A. Galstyan. Diffraction and diffraction radiation // UFN. 2000, v. 170, № 8, p. 809-830.

4. F.G. Bass, V.M. Yakovenko. Theory of radiation harge passing through electrically inhomogeneous media // UFN. 1965, v. 86, issue 2, p. 189-230.

5. V.A. Balakirev, G.L. Sidel'nikov. Excitation of electromagnetic pulse by relativistic electron bunch // Technical Physics. 1999, v. 44, № 10, p. 1209-1214.

6. V.A. Balakirev, N.I. Gaponenko, A.M. Gorban, et al. Excitement TEM-horn antenna by impulsive relativistic electron beam // Problems of Atomic Science and Technology. Series "Plasma Physics". 2000, № 3, p. 118-119.

7. V.A. Balakirev, I.N. Onishchenko, D.Yu. Sidorenko, G.V. Sotnikov. Wide-band emission of relativistic electron bunch in semi-infinite waveguide // Zhurnal Tekhnicheskoj Fiziki. 2002, v. 72, № 2, p. 88-95 (in Russian).

8. L. Felsen, N. Varcuvitz. Radiation and scattering of waves. Moscow: "Mir", 1973, v. 1, 548 p.

9. A.P. Prudnikov, Yu.A. Brychkov, O.I. Marichev. Integrals and series. Special functions. Moscow: "Nauka", 1983, 748 p.

10. L.A. Weinstein. Theory of diffraction and method of factorization. Moscow: "Soviet radio", 1966, $431 \mathrm{p}$.

Article received 14.06.2021

\section{ПЕРЕХОДНОЕ ВОЗБУЖДЕНИЕ ИДЕАЛЬНО ПРОВОДЯЩЕГО ЦИЛИНДРА РЕЛЯТИВИСТСКИМИ ЭЛЕКТРОННЫМИ СГУСТКАМИ}

\section{В.А. Балакирев, И.Н. Онищенко}

Рассмотрено переходное излучение релятивистского электронного сгустка, возникающее при его столкновении с поверхностью бесконечного идеально проводящего цилиндра. Электронный сгусток движется перпендикулярно поверхности цилиндра. Получены и исследованы выражения для напряженности поля электромагнитного излучения в волновой зоне.

\section{ПЕРЕХІДНЕ ЗБУДЖЕННЯ ІДЕАЛЬНО ПРОВІДНОГО ЦИЛІНДРА РЕЛЯТИВІСТСЬКИМИ ЕЛЕКТРОННИМИ ЗГУСТКАМИ}

\section{В.А. Балакірєв, І.М. Оніщенко}

Розглянуто перехідне випромінювання релятивістського електронного згустка, що виникає при його зіткненні з поверхнею нескінченного ідеально провідного циліндра. Електронний згусток рухається перпендикулярно поверхні циліндра. Отримано і досліджено вирази для напруженості поля електромагнітного випромінювання в хвильової зоні. 